to combine adult and paediatric practice (eg, the chapters on assessment and on intestinal failure-associated liver disease). I would not be so unkind as to describe it from the perspective of the curate faced with the dodgy egg, but I did find it somewhat unsatisfactory. It is good as it prompts both groups of specialists to consider problems more particular to the other, but these chapters seem to lose focus in the process. I was disappointed to find no mention of the random urine sodium in assessment of the intestinal failure patient-in my view a hugely helpful simple investigation-but perhaps its absence is symptomatic of its neglect in the literature as a whole.

An important portion of the book revolves around intestinal transplantation (16 of the 42 chapters), and there are many gems therein. It is helpful to have the surgical approaches summarised, but these are of most practical relevance to those in transplant centres, and I think most potential readers will be more concerned with the prior strategies on indications and referral, and with the postoperative aspects in patients who may be seen once they are released from hyperspecialist care. Accordingly I was disappointed with the chapter on long-term management as the opportunity to provide a hit-list of things that must and must not be done has been missed. This screams out for a secure algorithm for the non-specialist centre faced with a sick (or indeed not so sick) transplant patient. Finally there are no transplant outcome data more recent than 2005-6, which is a shame for such a rapidly moving field; I had hoped that Professor Grant might have given a sneak preview of more recent results.

On balance I am very positive about the book, consider it very good value for money and would strongly recommend it to all units dealing with intestinal failure. Some opportunities have been missed, and I am sure these will be dealt with in the future editions that are likely to emerge. It scores over the Nightingale book in its handling of transplantation, but this is mainly the consequence of the 7 years that separate the two volumes. Personally I am pleased to have both!

\section{Alastair Forbes}

Correspondence to: Professor A Forbes, Department of Gastroenterology and Nutrition, University College Hospital, 235 Euston Road, London NW1 2BU, UK; a.forbes@ucl.ac.uk

\section{Endoscopy in a new light...}

Edited by J Cohen. Published by Blackwell, Oxford, 2007 pp 317, hardcover with DVD, f110. ISBN 9781405158862

Narrow band imaging (NBI) comes as standard with the latest version of the hardware sold by the UK's most popular supplier of endoscopy equipment, but how many UK gastroenterologists know the theory of the new modality, how to use it, the appearances of even common lesions and the potential benefits for patients? This book attempts to bring the experience of the (mostly Japanese) front runners in NBI to a more general audience.

NBI uses restricted parts of the spectrum of white light to enhance the vessel pattern and superficial detail of mucosal lesions, allowing the diagnosis of early lesions such as cancers and dysplasia, so poor in current UK endoscopic practice. It is easy to use in narrow tubular organs such as the oesophagus and colon where light intensity is relatively high; light levels are lower in the more capacious stomach, reducing the value of NBI as a screening tool, though it still appears to be of value in detailed study of lesions first suspected by white light endoscopy. It is easy to deploy at the touch of a button, making it more practical than chromoendoscopy. There is a lot to learn about interpretation of the images where haemoglobin is shown in brown or green depending on the depth and size of the containing vessel, and the importance of the microvascular pattern. The common artefacts, due to poor bowel preparation for example where faeces is rendered as red, take some adjustment in the brain of the endoscopist. There are also more related acronyms and more Japanese classifications to take on!

The utility of NBI is not yet well supported by peer-reviewed publications, and as this book makes clear, it is complementary to a variety of other modalities including high-resolution endoscopy (HRE), magnification and chromoendoscopy which are not yet routine in UK endoscopy units.

The chapters explaining the basis of NBI and its potential applications are in quite good English, but some parts have not translated well from the original Japanese articles. The book contains a large series of good-quality endoscopic photos which could be better arranged, however. The illustrations with each chapter are printed at the end of the chapters, rather than integrated in the text where they would be easier to relate. The atlas in the second half of the book presents white light, NBI and magnification and chromoendoscopy images of lesions, but not always on the same page which is slightly irritating. The text and images also come on an included searchable DVD containing goodquality still images from the book and 55 video clips. The perfection of the Japanese clips is remarkable and the videos are very helpful in understanding the NBI appearances, though a number of the sequences are irrelevant to the subject of the book and seem to have been included as "stocking fillers".

Endoscopy now has to compete with other non-invasive imaging techniques. NBI, HRE and other new intraluminal imaging modalities are likely to give endoscopy a sensitivity unmatched by any other technique, and combined with its therapeutic potential will contribute to the development and survival of endoscopy. The benefits to patients of this increased sensitivity will need to be shown, but serious endoscopists will want to assimilate the contents of this publication, recognising that the study of NBI has much potential.

\section{Peter D Fairclough}

Correspondence to: Dr P Fairclough, Endoscopy Unit, The Royal London Hospital, London E1 1BB, UK; peter.f@ pobox.com

\section{CORRECTIONS}

doi:10.1136/gut.2007.137703corr1

J-H Jang, W Moritz, R Graf, et al. Preconditioning with death ligands FasL and TNF- $\alpha$ protects the cirrhotic mouse liver against ischaemic injury. Gut 2008;57:492-9. The authors would like to add one further source of funding: EMDO stiftung, Zurich, Switzerland (to J-H Jang and P-A Clavien).

\section{doi:10.1136/gut.2007.129296corr1}

Y Motomura, J-E Ghia, H Wang, et al. Enterochromaffin cell and 5-hydroxytryptamine responses to the same infectious agent differ in Th1 and Th2 dominant environments. Gut 2008;57:475-81. There is an error in the key for figure 5: solid bars represent SCID+ Th2 and open bars represent SCID+ Th1 